\title{
Treinamento físico: considerações práticas e científicas
}

\author{
Hamilton ROSCHEL* \\ Valmor TRICOLI* \\ Carlos UGRINOWITSCH* \\ *Escola de Educa- \\ ção Física e Esporte, \\ Universidade de São \\ Paulo.
}

\section{Resumo}

0 treinamento físico é uma importante área de atuação profissional da Educação Física e do Esporte. Ela tem por objetivo precipuo, a melhoria do desempenho físico-esportivo através da aplicação de um processo organizado e sistemático composto por exercícios físicos. Nos últimos anos, os progressos tecnológicos e nos métodos de investigação científica nas diferentes subáreas relacionadas ao treinamento físico trouxeram um avanço significativo na obtenção deste objetivo. Neste artigo será discutido, do ponto de vista acadêmico-científico e também da prática profissional, o estado da arte do conhecimento associado à avaliação do treinamento, ao controle da carga de treinamento, aos modelos de organização da carga de treinamento e ao desenvolvimento das capacidades motoras. Esperamos que ao final, o leitor possa ter um bom entendimento destes diferentes componentes, como eles contribuem para a modificação do desempenho motor e como aplicá-los para a elaboração, implementação, avaliação e reformulação de programas de treinamento físico.

UnITERMOS: Treinamento físico; Desempenho esportivo; Atuação profissional.

\section{Introdução}

O entendimento do termo treinamento é, sem dúvida, bastante amplo, tendo sido empregado nas mais variadas áreas científicas e profissionais. Treinamento é caracterizado como um processo repetitivo e sistemático composto de exercícios progressivos que visam o aperfeiçoamento do desempenho. Neste sentido, o treinamento físico pode ser compreendido como um processo organizado e sistemático de aperfeiçoamento físico, nos seus aspectos morfológicos e funcionais, impactando diretamente sobre a capacidade de execução de tarefas que envolvam demandas motoras, sejam elas esportivas ou não (Barbanti, Tricoli \& Ugrinowitsch, 2004).

As origens das bases teóricas do treinamento físico não são recentes. Grandes pensadores da antiguidade já propunham teorias acerca do treinamento em seus trabalhos. Galeno de Pérgamo (século segundo d.C.) já ensaiava sobre conceitos que se assemelham ao que se entende atualmente por periodização. No seu tratado "Preservação da Saúde", ele discorre sobre sequências de treinamento para o desenvolvimento específico da força rápida, passando pelo uso de exercícios que priorizavam a força em detrimento da velocidade, culminando com o uso de exercícios intensos que combinavam as duas capacidades motoras. Já Filóstrato, o Atenienese, propunha também no século segundo d.C., um período preparatório para as competiçôes Olímpicas, inclusive com a sugestão de um período destinado à preparação específica em um centro de treinamento um mês antes da competição (GARDINER, 1930; ISSURIN, 2010).

Embora os exemplos acima ilustrem uma preocupação já bastante antiga com o treinamento físico voltado para o desempenho, não foi até poucas décadas atrás que a ciência do esporte realmente experimentou um avanço significativo no seu corpo de conhecimento. A ciência do esporte pode ser entendida como uma área multidisciplinar preocupada com a compreensão e aperfeiçoamento do desempenho físico-esportivo humano (BISHOP, 2008). Ainda segundo o autor, a ciência do esporte pode ser vista como o processo científico usado para guiar a prática esportiva e, em última análise, melhorar o desempenho físico. Neste sentido, a ciência do esporte tem cada vez mais contribuído para o aperfeiçoamento dos programas de treinamento físico-esportivo, através principalmente de um aumento na qualidade do treinamento proposto. 
Assim, podemos didaticamente dividir os estudos relacionados ao treinamento físico-esportivo em quatro grandes áreas: 1) avaliação do treinamento; 2) controle do treinamento; 3) modelos de organização da carga de treinamento e 4) desenvolvimento das capacidades motoras. Entender a importância de cada uma dessas áreas para a maximização do rendimento físico-esportivo é fundamental para o profissional, pois as exigências do esporte moderno tanto em relação às

\section{Desenvolvimento}

\section{Avaliação do treinamento}

O desempenho físico é considerado parte integral do esporte e sua avaliação constitui um aspecto fundamental na análise da eficácia dos processos de treinamento empregados ao longo de uma temporada (Kiss \& BöHme, 2003).

Contudo, para que a avaliação seja efetiva, é necessário que os aspectos mais importantes para o rendimento físico-esportivo em uma determinada modalidade esportiva sejam investigados. É amplamente aceito que na vasta maioria das modalidades esportivas não há uma única capacidade motora que caracterize a modalidade, mas sim, um conjunto de capacidades que é determinante para um elevado rendimento esportivo. Quanto mais complexa for a estrutura da atividade esportiva (ex: corrida de $100 \mathrm{~m}$ vs. basquetebol), mais importante é a interação entre as capacidades motoras. Por isso, a avaliação deve compreender o diagnóstico de todas as capacidades envolvidas na modalidade esportiva alvo e, se possível, em condições que simulem a prática da atividade (Currell \& Jeukendrup, 2008). Desta forma, muitas pesquisas têm se dedicado ao desenvolvimento de métodos que permitam identificar as capacidades motoras envolvidas em diversas modalidades esportivas. Além dos métodos mais usuais encontrados na literatura, uma contribuição muito grande vem sendo dada por sistemas de vídeo, os quais têm sido utilizados para descrever as atividades motoras realizadas durante as competições esportivas de modalidades como o futebol, o basquetebol e o voleibol (BEN ABDELKRIM, Castagna, El FazAa \& El Ati, 2010; MoHr, Krustrup, Andersson, Kirkendal \& Bangsbo, 2008; Sheppard, GabBetT \& Stanganelli, 2009).

Adicionalmente, grande atenção tem sido dada à elaboração de testes que sejam válidos (i.e. o protocolo assemelhe-se o tanto quanto possível ao desempenho cargas de treinamento, quanto ao número de competições por temporada, faz com que os atletas estejam mais predispostos a lesões e estados de sobretreinamento, nos quais o rendimento esportivo é negativamente afetado.

O objetivo desse ensaio é, portanto, apresentar uma breve discussão sobre o estado da arte no que concerne ao conhecimento em cada uma destas áreas ligadas ao treinamento físico-esportivo e suas aplicações práticas.

esportivo que está sendo simulado), tenham uma elevada reprodutibilidade (i.e. os resultados são consistentes ao longo do tempo) e sejam sensíveis para detectar mesmo pequenas alteraçôes no desempenho provocadas pelo processo de treinamento (CURRELL \& Jeukendrup, 2008; Hopkins, 2000).

A avaliação da força motora, por exemplo, tem sido amplamente utilizada tanto para inferências sobre o desempenho esportivo quanto para a obtenção de parâmetros para a prescrição do treinamento. Em relação ao desempenho esportivo, podemos citar estudos que identificaram o tempo, a magnitude e a direção da força aplicada nas diversas fases da corrida de velocidade (Mero, Komi \& Gregor, 1992). Foram também investigadas as forças produzidas na decolagem do flic-flac e do salto mortal (PAYNE \& BARKER, 1975), além do giro gigante nas argolas, e na ginástica olímpica (NissineN, 1983). No tocante à prescrição do treinamento, o teste de força dinâmica máxima (1 RM) ilustra bem o avanço tecnológico na avaliação do treinamento. Embora este teste não seja específico à grande maioria das modalidades esportivas, ele pode ser usado para avaliar o perfil inicial de um determinado atleta antes do início do programa de treinamento, fornecendo parâmetros para a prescrição do treino de força deste atleta e posteriormente verificando a eficácia do programa elaborado. Os dinamômetros isocinéticos também são amplamente utilizados para a avaliação da força (Parcell, Sawyer, Tricoli \& Chinevere, 2002). Tais equipamentos apresentam elevada reprodutibilidade entre avaliações, além de fornecerem estimativas do torque, do trabalho e da potência produzidos na tarefa motora (UGRINOWITSCH, BARBANTI, GONÇALVES $\&$ Peres, 2000). Contudo, eles têm uma limitação muito grande, já que normalmente só utilizam movimentos monoarticulares e com uma velocidade de movimento constante (ABERNETHY, WiLSON \& 
LogAn, 1995). Células de carga também têm sido utilizadas para avaliar a força muscular máxima em condições isométricas (i.e. em que não há movimento externo aparente). Uma informação muito interessante obtida nos testes isométricos balísticos, nos quais a força é produzida o mais rapidamente possível, é a determinação da taxa de desenvolvimento de força (TDF) (RICARD, UGRINOWITSCH, PARCELL, Hilton, Rubley, Sawyer \& Poole, 2005). A TDF é considerada uma variável importante para o desempenho físico-esportivo, já que indica a capacidade do indivíduo aplicar força o mais rapidamente possível contra uma determinada resistência.

Variaçōes do salto vertical (em semi-agachamento, com contra-movimento e em profundidade) são comumente empregadas em avaliações indiretas da potência muscular de membros inferiores. Esta é normalmente inferida pela altura do salto, ou seja, quanto maior a altura, maior a produção de potência. Porém, os avanços tecnológicos permitem hoje avaliações mais completas, contemplando outras variáveis de interesse para a compreensão do desempenho. Por exemplo, o uso de plataformas de força permite avaliar tanto o efeito de diferentes históricos de treinamento quanto de modelos de treinamento na mecânica externa do salto vertical, a fim de selecionar os meios e métodos de treinamento mais eficientes para aumentar a potência de acordo com as características da modalidade esportiva em questão (UGrinowitsch, Tricoli, Rodacki, BatisTA \& RICARD, 2007). Já a combinação dos dados provenientes de plataformas de força e de filmagens permite quantificar, através de modelos matemáticos, as forças internas que incidem sobre o aparelho locomotor durante o movimento, a velocidade angular das articulações envolvidas no salto, a velocidade no instante da decolagem e a sincronia dos movimentos articulares entre outros parâmetros de interesse.

Além das técnicas descritas, potenciômetros lineares têm sido conectados a barras de aparelhos de musculação, como o "Smith machine", para identificar a produção de potência em exercícios como o agachamento e o supino. Através das mudanças de posição da barra no tempo e da magnitude da carga deslocada, é possível calcular a produção de potência durante os exercícios (Bosco, Belli, Astrua, Tihanyi, Pozzo, Kellis, Tsarpela, Foti, Manno \& TranQUILLI, 1995). Esses cálculos permitem determinar o efeito e as características de diferentes modelos de treinamento na produção de potência (LAMAS, BAtista, Fonseca, Pivetti, Tricoli \& Ugrinowitsch, 2010; Lamas, Drezner, Tricoli \& Ugrinowistch,
2008) e a zona de treinamento na qual a produção de potência é maximizada (TojI, Suei \& KANEKO, 1997).

A avaliação das demais capacidades motoras também se beneficiou dos avanços tecnológicos. A avaliação da flexibilidade, comumente realizada de forma inespecífica através do teste de sentar e alcançar, pode hoje ser feita em todas as articulações e eixos de movimento com o uso de flexímetros, bem como em condições muito mais próximas $\mathrm{da}$ especificidade inerente ao gesto esportivo, com o uso de eletrogoniômetros multi-axiais (i.e. medem a amplitude de movimento em mais de uma direção) telemétricos e de dimensões reduzidas (http://www. pennyandgiles.com).

O mesmo pode-se dizer da capacidade e potência aeróbia. O desenvolvimento de sistemas de análise de gases portáteis com telemetria tem possibilitado a avaliação do consumo de oxigênio em condições mais próximas da prática da modalidade esportiva, como nas provas de atletismo e em jogos coletivos, entre outros. Já a potência anaeróbia tem sido inferida a partir da mensuração do trabalho máximo realizado em um determinado tempo. Como as medidas de trabalho têm sido alvos de avanços tecnológicos, as inferências sobre a potência anaeróbia têm se beneficiado deste fato.

O uso de técnicas como as de biópsias musculares e de biologia molecular também pode ser destacado como um avanço tecnológico importante no estudo das adaptaçôes agudas e crônicas aos diferentes modelos de treinamento. É possível, por exemplo, avaliar qual modelo de treinamento é mais efetivo para promover adaptações relacionadas ao funcionamento do metabolismo aeróbio (e.g. biogênese mitocondrial, vascularização, mudança no fenótipo da fibra muscular). Embora estes modelos de avaliação não façam nenhuma mensuração direta sobre o desempenho físico-esportivo, os dados oriundos destas podem, em última análise, serem usados a favor da melhora do desempenho a partir da resposta fisiológica produzida pelos mesmos. Pode-se ainda, avaliar como diferentes modelos de treinamento de força afetam a atividade dos processos intracelulares que desencadeiam alteraçôes morfológicas, como a hipertrofia muscular (ROSCHEL, UGRINOWISTCH, Barroso, Batista, Souza, Aoki, Siqueira-Filho, Zanuto, Carvalho, Neves, Mello \& Tricoli, 2011), permitindo assim, detectar os melhores modelos de treinamento para aumento da massa muscular.

Outro aspecto fundamental da avaliação no esporte diz respeito à avaliação do jogo. As modalidades esportivas coletivas apresentam, na sua maioria, mudanças de situação frequentes, impondo um 
alto grau de imprevisibilidade. Essa característica, onde os comportamentos e ações estão sujeitas a aleatoriedade e variabilidade, exige do praticante uma grande capacidade de adaptação, o que implica que o desempenho depende amplamente da capacidade de tomada de decisão, enfatizando a importância do componente tático. Neste sentido, a análise de jogo apresenta vantagens importantes para o processo de treinamento, caracterizados, por exemplo: 1) pelo rápido fornecimento de informaçōes para uma análise em tempo real, que permite a tomada de decisóes durante o jogo; 2) pela criação de uma base de dados de caracterização das ações táticas de ataque e defesa dos adversários que norteiam processos de treinamento específicos e 3) pela a análise tática e técnica individuais e de grupo, permitindo intervenções sobre a estratégia de uma determinada equipe (CASTRO \& MESQUITA, 2008; Lamas, Rose Junior, Santana, Rostaiser, NegretTI \& UGRINOWITSCH, 2011).

Vários sistemas informatizados específicos para esta análises podem ser encontrados atualmente, o que tem facilitado amplamente o uso tanto de análises técnicas (http://www.dartfish.com/en/ software/index.htm) quanto táticas no fornecimento de "feedback" em tempo real na competição, como também na orientação do treinamento de diferentes modalidades esportivas. Com essa tecnologia de avaliação é possível avaliar desde indicadores de desempenho, como o número de ataques certos realizados com um golpe específico no caratê ou no tênis de mesa, ou o número de finalizaçôes de uma determinada região da quadra de uma equipe de basquetebol, até os padrōes táticos ofensivos e defensivos adotados por equipes de modalidades de invasão como o futebol, futsal e handebol.

\section{Controle do treinamento}

O processo de treinamento pode ser simplificado em uma relação entre "dose" e "resposta" (LAMBERT \& BorReSEn, 2010). Nesta perspectiva, a resposta estaria associada à mudança no desempenho ou a alteração de algum parâmetro fisiológico, decorrente de uma determinada dose de treinamento, a qual pode ser definida como o estresse fisiológico imposto ao corpo do atleta por uma determinada carga de treinamento. Esta tem sido comumente chamada de carga interna de treinamento. Até muito recentemente, a prescrição do treinamento era feita de maneira muito intuitiva, fazendo com que a experiência do treinador fosse determinante para o sucesso do atleta (BORRESEN \& LAMBERT, 2009). Isso certamente representa uma limitação quando se pretende estabelecer uma relação entre o treinamento prescrito, o que isso representa para o atleta em termos de demanda fisiológica e as mudanças no desempenho decorrentes do processo de treinamento (LAMBERT \& BORRESEN, 2010). Frente a isso, tanto os técnicos e preparadores físicos quanto os cientistas têm buscado determinar parâmetros que sejam capazes de controlar a carga de treinamento imposta aos atletas.

Inicialmente, o controle da carga de treinamento era feito apenas através do controle da chamada "carga externa". Parâmetros como o volume total (número de repetiçõos x sobrecarga utilizada, quilômetros percorridos e número de tiros realizados), trabalho realizado (força produzida x distância percorrida), ou o tempo sob-tensão, entre outros, eram os únicos indicadores considerados no controle do treinamento (McBride, McCaulley, Cormie, Nuzzo, Cavill \& TRIPlETT, 2009). Contudo, esse modelo não consegue representar a repercussão mecânica e fisiológica que uma determinada carga de treinamento impóe sobre o organismo. O problema da quantificação adequada da carga de treinamento se estende além do campo prático. Nas pesquisas em esporte, um método mais válido e confiável de avaliação da carga de treinamento é de extrema importância nas investigações acerca da eficácia de um determinado modelo de treinamento e da relação entre carga de treino e desempenho (Bishop, 2008; Borresen \& LAMBerT, 2009).

Dentre os parâmetros fisiológicos de mensuração da carga interna de treinamento, podemos destacar a avaliação do consumo de oxigênio $\left(\mathrm{VO}_{2}\right)$, a qual tem sua validade e reprodutibilidade demonstradas em laboratório. Contudo, a sua aplicação em campo durante as competições ou até mesmo treinamentos é pouco prática ou até não factível, limitando o seu uso nessas condições. Além disso, o uso da avaliação do $\mathrm{VO}_{2}$ não é adequado em atividades intermitentes ou em exercícios supra-máximos. A concentração sanguínea de lactato também tem sido utilizada como um marcador da intensidade do treinamento. Entretanto, este método é influenciado por vários fatores, como a dieta, o tipo de exercício utilizado no treinamento e o estado de treinamento do indivíduo. Assim, ambos os métodos, embora tenham sua validade e utilidade no ambiente laboratorial, são pouco adequados para o monitoramento em campo da carga de treinamento.

Métodos mais simples, como os questionários e diários/recordatórios de treino, têm sido usado como um método bastante prático de monitoramento da carga de treino. Contudo, estes métodos apresentam baixa reprodutibilidade e validade concorrente se comparados aos métodos laboratoriais (SHEPHARD, 2003). 
A frequência cardíaca (FC) também tem sido utilizada como parâmetro de carga interna, dada a proposição de uma relação linear entre esta e a taxa de incremento de trabalho. Como a FC varia de acordo com a idade e o estado de treinamento, entre outros fatores, a FC de reserva é mais utilizada para prescrição da intensidade do treinamento (FC $\left.=\mathrm{FC}_{\max }-\mathrm{FC}_{\text {repouso }}\right)$. Contudo, apesar de ela poder ser mensurada de forma acurada durante o exercício (através de monitores de FC), ela não é um bom indicador para atividades intermitentes, nas quais a relação linear entre trabalho e FC deixa de ocorrer, afetando assim, a sua eficácia como marcador do estresse fisiológico imposto pelo exercício.

A percepção subjetiva do esforço (PSE) é uma forma alternativa de quantificação da carga interna do treinamento. Esta é baseada na assunção de que o atleta tem uma capacidade inerente de perceber o esforço ao qual seu corpo está sendo submetido e ajustar a intensidade do mesmo baseado nessa percepção. Contudo, a correlação entre PSE e parâmetros como a FC, por exemplo, parece ser razoável apenas em exercícios realizados em intensidade constante e em um estado de "equilíbrio fisiológico", limitando sua aplicação em outras formas de exercício.

Apesar dos avanços obtidos no controle da carga de treinamento através da utilização da FC e da PSE, esses indicadores ainda parecem ineficientes em determinar a carga de uma sessão de treinamento. Determinar o efeito de uma sessão de exercícios no organismo é extremamente importante, pois há que ser considerado que atletas treinam vários dias na semana e, muitas vezes, mais de uma vez por dia. Caso um atleta tenha sessões sucessivas de treinamento que produzam um estresse excessivo, isso fará com que ele esteja mais susceptível a lesóes. A partir desta limitação, a PSE da sessão de treino foi proposta por (Foster, Daines, Hector, Snyder \& Welsh, 1996; Foster, Florhaug, Franklin, Gottschall, Hrovatin, Parker, Doleshal \& Dodge, 2001). Neste caso, a PSE é um reflexo não só do estresse fisiológico ao qual o sujeito foi submetido, mas também da duração deste esforço, sendo calculada pela multiplicação da PSE (1-10) pela duração em minutos, da sessão de treino. Embora este modelo apresente uma melhor correlação com outros marcadores de carga interna, ele ainda tem suas limitaçõos, em especial quando utilizado no treinamento de força. Apesar do exposto, um estudo recente demonstrou que a PSE determinada pelos técnicos era similar àquela determinada pelos atletas, evidenciando que técnicos experientes conseguem ter uma boa ideia da carga de treino (Wallace, Slattery \& Coutts, 2009). Por outro lado, nas modalidades esportivas que envolvam contato físico intenso, os danos musculares resultantes deste contato podem influenciar a PSE, criando um viés na avaliação (TAKARADA, 2003).

Outras formas de avaliação da carga de treinamento têm sido propostas. Alguns marcadores bioquímicos têm sido usados na tentativa de determinar o estresse produzido por uma determinada carga de treinamento. De fato, pode-se encontrar estudos com treinamento de força, por exemplo, que têm demonstrado que a intensidade do exercício influência a secreção de cortisol (KRAEMER, Hakkinen, Newton, Nindl, Volek, McCormick, Gotshalk, Gordon, Fleck, Campbell, Putukian \& Evans, 1999; Mulligan, Fleck, Gordon, Koziris, Triplett-McBride \& Kraemer, 1996). Ainda, a secreção de cortisol parece ser sensível a variações na carga de treinamento de força em jogadoras de basquete de nível internacional (NUNES, CREWTHER, Ugrinowitsch, Tricoli, Viveiros, De Rose Junior $\&$ АокI, 2011). Contudo, outros estudos mostram que ao variarmos tanto a intensidade quanto o volume de treinamento, nenhuma resposta hormonal é verificada (Hough, Papacosta, Wraith \& Gleeson, 2011; Roschel, Barroso, Batista, Ugrinowitsch, Tricoli, Arsati, Lima-Arsati, Araujo \& Moreira, 2011; Smilios, Pilianidis, Karamouzis \& TokmakiDIS, 2003). Estas discrepâncias entre os estudos têm revelado que a variação hormonal frente ao exercício é multifatorial, sendo influenciada pela hora do dia, estado alimentar e tempo de coleta após o exercício. Alternativamente, a avaliação da imunoglobulina A ( $\operatorname{IgA}$ ) tem sido utilizada como ferramenta na avaliação da carga interna de treinamento. A IgA parece responder à intensidade do exercício, sendo um importante marcador da função imunológica do atleta, estando associada com a incidência de infecções do trato respiratório superior (Ahtiainen, PaKarinen, Alen, Kraemer \& HaKkinen, 2005; Nieman, 1994).

Atualmente, não há uma forma de quantificação acurada que permita a prescrição de um padrão, duração e intensidade do treinamento necessária para induzir adaptações fisiológicas específicas. Somado a isto, está o fato que os indivíduos parecem se adaptar de maneiras distintas a um mesmo estímulo de treino. Adicionalmente, as técnicas de monitoramento não têm se preocupado no acompanhamento da forma esportiva ao longo do programa de treinamento. Por outro lado, o esporte atual não permite a obtenção da forma esportiva, pois os atletas têm que manter um elevado rendimento esportivo (sub-máximo, 
mas ótimo) por longos períodos de tempo, fazendo com que as técnicas que monitoram estados de "overreaching" e "overtraining" ganhem importância. Finalmente, as diferentes técnicas deveriam progredir para que o monitoramento do treinamento deixasse de ser apenas físico e entrasse no campo técnico, tático e psicológico.

\section{Modelos de organização da carga de treinamento}

A partir da década de 50 do século passado os modelos de organização da carga de treinamento começaram a ganhar muita popularidade. $\mathrm{O}$ sucesso dos atletas da antiga União Soviética foi, por muitos anos, o cartão de visita da teoria da periodização do treinamento esportivo (Matveev, 2001). A periodização preconizava que os atletas deveriam ter longos períodos de preparação (i.e. entre três e oito meses) antes de participarem das competições alvo. Ainda, dois terços do tempo do chamado período preparatório deveriam ser dedicados a exercícios de preparação geral, com baixo nível de especificidade. Os Jogos Olímpicos de Moscou em 1980 trouxeram uma importante alteração na organização dos programas de treinamento. A guerra fria estava no seu auge e tinha como objetivo implícito o embate entre os sistemas políticos dominantes na época - Capitalismo e o Socialismo. Para que a União Soviética sobrepusesse os seus adversários, o Prof. Yuri Verkhoshansky desenvolveu um novo modelo de "periodização" chamado de treinamento em blocos (VERKHOSHANSKY, 1986). A grande evolução desse sistema em relação à periodização de Matveev foi a utilização de parâmetros biológicos para orientar o treinamento dos atletas. O seu sistema preconizava a ocorrência do "overreaching" no bloco inicial do treinamento. A queda de rendimento decorrente deste processo deveria ser recuperada nos blocos posteriores. Na verdade, o Prof. Verkhoshansky preconizava que quanto maior a queda de rendimento no bloco inicial, maior seria a elevação posterior do desempenho. Apesar da escassez de evidência científica na aplicação deste tipo de periodização, as contribuições do Prof. Verkhoshansky à teoria do treinamento foram muito importantes.

Deve ser lembrado que muitos dos primeiros modelos de periodização do treinamento requeriam um período preparatório longo. Assim, para a aplicação no esporte moderno, eles necessitam de adaptaçôes para se adequarem ao elevado número de competições ao longo do ano. Além disso, há muito poucos estudos dedicados à comparação entre os modelos tradicionais e os contemporâneos de periodização do treinamento, sendo que parece não haver diferenças substanciais no rendimento entre eles (MOREIRA, 2006).

Outros modelos de periodização vêm sendo propostos nas últimas décadas (ForTeZA, 2006; NAVARRO, 1998). Porém, da mesma forma que com os modelos de Matveev e Verkhoshansky, a implementação de modelos de pesquisa que comparem esses modelos é muito difícil, já que há a necessidade de se utilizar atletas com bom nível competitivo e que possam treinar de diferentes formas, dentro do mesmo período de tempo, o que nem sempre é possível no cenário científico.

Estas diferentes formas de organização do treinamento parecem afetar também o desempenho de força máxima e de potência muscular. Alguns achados preliminares (citação de dados não publicados) indicam que mais importante do que a periodização do treinamento é a correta seleção do seu conteúdo (i.e. exercícios e capacidades motoras a serem trabalhadas), pois diferentes modelos de organização produziram ganhos de força, potência e velocidade semelhantes.

De maneira mais simplificada, a organização temporal do treinamento de força é mais facilmente implementada e já foi testada em alguns estudos. $\mathrm{O}$ interesse pela organização do treinamento da força é justificado pela importância que essa capacidade tem para um elevado rendimento esportivo (LAMAS et al., 2010; Tricoli, Lamas, Carnevale \& UGrinowitsch, 2005). Três modelos básicos de organização das cargas de treinamento de força são reportados na literatura. Esses modelos são chamados de não-periodizados, periodizados lineares e periodizados não-lineares. Nos modelos não-periodizados não há variações no volume e na intensidade relativa (\% de $1 \mathrm{RM}$ ) dos exercícios, nos modelos periodizados lineares há uma diminuição do volume e um aumento da intensidade ao longo do período de treinamento e, nos modelos periodizados não-lineares, há variações semanais ou diárias na carga de treinamento.

A comparação entre esses modelos tem trazido resultados bastante controversos, pois a maioria dos estudos verificou apenas os ganhos de força, desconsiderando as adaptações neurais e morfológicas decorrentes do processo de treinamento. Uma meta-análise indicou que o modelo não linear parece ser mais eficiente que os modelos não periodizado e periodizado linear (RHEA, 2004).

Vale ressaltar que os estudos dedicados a avaliação de diferentes modelos de periodização do 
treinamento precisam ser expandidos também na dimensão da preparação física. Sabe-se que o desenvolvimento simultâneo das capacidades motoras, força e resistência dentro de um mesmo período de treinamento pode prejudicar as adaptações produzidas na força e na potência musculares (DE SouZA, Rosa, Pires, Wilson, Franchini, Tricoli \& UgriNOWitsch, 2011; De Souza, Tricoli, Franchini, Paulo, Regazzini \& Ugrinowitsch, 2007). Ainda, a realização de exercícios de flexibilidade estática antes de exercícios de força (Bacurau, Monteiro, Ugrinowitsch, Tricoli, Cabral \& Aoki, 2009) e resistência aeróbia (WiLSON, HoRnbuCKLe, KIM, Ugrinowitsch, Lee, Zourdos, Sommer \& Panton, 2010) parece afetar a capacidade de produção de força e a economia de corrida, respectivamente. Como o rendimento esportivo de inúmeras modalidades esportivas depende do treinamento de diversas capacidades motoras dentro de um mesmo período de treinamento, os pesquisadores deveriam investigar os efeitos da interação do desenvolvimento/treinamento de diversas capacidades motoras de maneira simultânea no desempenho esportivo. Além disso, pouco se sabe a respeito dos efeitos da periodização e do treinamento simultâneo de diferentes capacidades motoras em componentes mais determinantes do rendimento esportivo, como o técnico e o tático.

\section{Desenvolvimento dascapacidadesmotoras}

O desenvolvimento das capacidades motoras de forma isolada é um tema muito abordado na literatura do treinamento. Vários estudos têm tentado identificar os métodos e os meios de treinamento mais apropriados para a maximização do desempenho para as diversas capacidades motoras. Contudo, esse tema é bastante amplo e controverso já que a resposta de um atleta a um determinado método de treinamento depende de um conjunto de fatores como a experiência (i.e. tempo de treinamento), a modalidade esportiva em questão, os métodos de treinamento utilizados anteriormente entre outros. Foge do escopo do presente manuscrito a discussão exaustiva de tais tópicos. Forneceremos, portanto, apenas alguns exemplos do que tem sido feito no âmbito científico acerca desta temática.

Os métodos de treinamento da flexibilidade podem ser genericamente divididos em três tipos: estático, dinâmico e combinados (e.g. alongamentocontração-relaxamento). Vários estudos têm relatado que esses três métodos são eficientes em aumentar o grau de flexibilidade. Em relação à duração do estímulo no treinamento de flexibilidade estática, o tempo total que o músculo permanece alongado parece ser mais importante que a duração de cada série (seis séries de 10 segundos vs. duas séries de 30 segundos) (Cipriani, Abel \& PirRWITZ, 2003). Na comparação entre o método estático ativo (i.e. realizado pelo próprio executante), estático passivo (i.e com auxílio externo) e combinado, os pesquisadores demonstraram que o método estático realizado por um ajudante externo foi mais eficiente em aumentar a flexibilidade dos flexores do joelho (DAvis, AshBY, McCale, McQuain \& Wine, 2005). Porém, outros pesquisadores não tem encontrado diferenças entre os métodos estático e combinado em estudos crônicos com seis semanas de treinamento (YUKTASIR \& KAYA, 2009). De maneira interessante, outro grupo de autores demonstrou que o tempo de contração dos músculos agonistas parece ser importante para os ganhos em flexibilidade ao se utilizar o método alonga-contrai-relaxa de maneira crônica (RowLANDS, Marginson \& LeE, 2003). Os autores observaram que o grupo que manteve a contração muscular por 10 segundos teve maiores ganhos do que o grupo que a manteve por cinco segundos. Os estudos que não encontraram uma maior eficiência do método alongacontrai-relaxa utilizaram contrações dos agonistas de cinco segundos, indicando que esse pode ter sido um fator que limitou os ganhos de flexibilidade (THACKer, Gilchrist, Stroup \& Kimsey Junior, 2004).

Entre os meios de treinamento para o desenvolvimento da velocidade podemos salientar o uso de corridas rápidas e curtas, corridas curtas em subidas, corridas com tração ou com colete de sobrecarga, exercícios pliométricos, saltos com sobrecarga, "overspeed" entre outros (WEINECK, 1999; ZAKHAROV \& GOMES, 2003). Em diferentes níveis, estes métodos têm se mostrado efetivos para o desenvolvimento da velocidade. De fato, demonstrou-se recentemente que o uso de treinamento pliométrico associado ao treino específico de tênis melhorou a velocidade de deslocamentos laterais e deslocamentos para frente e para trás em distâncias curtas $(4 \mathrm{~m})$, na aceleração em movimentos laterais para a rebatida no tênis e na altura de salto em tenistas jovens do sexo masculino (SALONIKIDIS \& ZAFEIRIDIS, 2008).

A força muscular é outra capacidade de grande importância para uma vasta gama de modalidades esportivas, merecendo grande atenção nos programas de treinamento. A força máxima é decisiva, por exemplo, em modalidades como o levantamento de peso, o arremesso no atletismo, os saltos, a corrida de velocidade, em algumas modalidades de luta e 
na ginástica artística. Já a força rápida ou potência é ainda mais abrangente, sendo de fundamental importância para as modalidades esportivas coletivas, nas modalidades de velocidade na natação e no atletismo, nas modalidades de luta, enquanto a resistência de força se faz importante em algumas modalidades como o ciclismo, o "ski cross-country", etc. (Platonov, 2008).

O desenvolvimento destas diferentes manifestações da força pode ser atingido através do treinamento específico com o uso de diferentes métodos e meios de treinamento. Destacam-se entre outros: a) o método isométrico funcional, que se utiliza de ações musculares estáticas em angulações pertinentes ao gesto esportivo em questão; b) o método isocinético, que faz uso de um dinamômetro isocinético, o qual controla a velocidade da contração muscular, tanto excêntrica quanto concêntrica, em intensidades máximas ou sub-máximas (assim como o isométrico, este método pouco se traduz em condiçōes reais de prática esportiva, indo contra o princípio da especificidade do treinamento) e c) o método dinâmico com o uso de ações excêntricas, concêntricas ou a combinação de ambas. A utilização de uma ação isolada pode ser interessante quando esta estratégia representar uma especificidade significativa com o gesto esportivo em questão. Por exemplo, para o treinamento do salto vertical para o bloqueio no vôlei de areia, dada a característica do piso, a força necessária para o salto vertical virá fundamentalmente dos componentes contráteis do músculo esquelético, já que a velocidade de transição entre a flexão e extensão dos joelhos é diminuída, fazendo com que a ação concêntrica deva ser enfatizada durante o treinamento (Tilp, Wagner \& Muller, 2008). A ação excêntrica, por sua vez, é sabidamente mais eficiente na indução dos ganhos de massa muscular (Higbie, Cureton, Warren \& Prior, 1996). Desta forma, pode-se optar por usar exercícios puramente excêntricos, ou de se enfatizar a ação excêntrica durante um dado exercício a fim de se aumentar a tensão na musculatura, induzindo maior ganho de hipertrofia. A combinação de ambas as ações musculares é, contudo, o método mais comumente utilizado, contemplando ambos os benefícios. $\mathrm{O}$ treino complexo é outro método bastante utilizado no desenvolvimento da potência muscular. Este método se baseia na combinação de cargas altas com cargas mais baixas, na tentativa de promover a potencialização pós-ativação (PPA). Embora alguns estudos tenham mostrado efeito positivo a partir do uso do treinamento complexo, (BATISTA, UGRINOWITSCH,
RosChel, LOTUFo, Ricard \& TRICOLI, 2007; FRENCh, Kraemer \& Cooke, 2003), o melhor modelo de atividade condicionante para induzir a PPA ainda não está estabelecido, já que esta parece depender de diferentes fatores como o estado de treinamento do indivíduo e a relação potencialização/fadiga.

É importante notar que os ganhos de força muscular são oriundos tanto de adaptações neurais quanto de adaptaçōes morfológicas. As diferentes fases das contribuiçōes neurais e hipertróficas na modificação da força muscular foram identificadas há décadas (Moritani \& De VRies, 1979). Estes pesquisadores foram os primeiros a demonstrar que os ganhos iniciais na força são oriundos de adaptações neurais (aumento no recrutamento e sincronização de unidades motoras, diminuição na co-contração da musculatura antagonista, aprendizagem do movimento) sendo que o aumento da massa muscular passa a contribuir de maneira significativa para a produção de força, somente após algumas semanas de treinamento.

Já o desenvolvimento da potência muscular requer máxima taxa de desenvolvimento de força (i.e. variação da força na unidade de tempo) durante as séries de exercícios (Hill-HaAs, Bishop, Dawson, GoOdMan \& Edge, 2007; Willardson \& BurketT, 2006). A literatura tem mostrado que o treinamento de potência frequentemente aplica a realização de 3-6 repetiçóes por série; intensidades de 30 a $60 \%$ de $1 \mathrm{RM}$; alta velocidade no movimento dos exercícios e 3-5 minutos de intervalo de descanso (CRONIN, McNair \& Marshall, 2000; Ratamess, Alvar, Evetoch, Housh, Kibler \& Kraemer, 2009). Contudo, uma vez que treinadores e profissionais do esporte preocupam-se com a especificidade das cargas em relação às modalidades esportivas, a manipulação do volume e o intervalo de descanso entre as séries dos exercícios desempenham um papel importante. Recentemente foi demonstrado (dados não publicados) que os treinadores podem reduzir com êxito o intervalo de descanso, para atender a uma demanda de tarefas específicas, e ainda manter a produção de potência desde que ocorra uma redução do volume nos exercícios realizados.

É fundamental salientar que a alteração dos níveis de força, seja pelo treinamento de força, seja pelo de potência, requer uma reorganização do controle do movimento para que a força seja revertida em desempenho no movimento desejado, enfatizando a importância do conteúdo específico na transferência do treinamento para a tarefa de campo (TRICOLI et al., 2005).

A resistência motora, por sua vez, é definida como a capacidade de sustentar uma determinada 
velocidade, ou produção de potência, pelo maior tempo possível (JONES \& CARTER, 2000). A melhoria dessa capacidade motora envolve adaptações nos sistemas pulmonar, cardiovascular e neuromuscular.

Notadamente, uma das principais adaptaçóes desejáveis ao se programar um treinamento de resistência é o aumento na potência aeróbia, medida através do $\mathrm{VO}_{2 \text { máx. }}$ Contudo, é importante destacar que outros fatores, além do $\mathrm{VO}_{\text {2máx }}$, explicam a variância no desempenho em provas de resistência de média e longa duração. Tem sido sugerido que a economia de corrida (entendida como o custo energético para manutenção de uma determinada velocidade de corrida) e a capacidade aeróbia (e.g. limiar aneróbio) são importantes determinantes do desempenho entre indivíduos com $\mathrm{VO}_{2 \text { máx }}$ similares (Bassett Junior \& Howley, 2000). De fato, um estudo demonstrou que o $\mathrm{VO}_{2 \text { max }}$ entre maratonistas quenianos e caucasianos era similar, contudo, o limar de lactato entre eles diferia significantemente (LARSEN, 2003). Quanto maior a velocidade de corrida correspondente ao limiar anaeróbio, maior a intensidade de exercício que um indivíduo consegue ter com uma predominância do metabolismo aeróbio. Com isso, os indivíduos com maior limiar anaeróbio, mas $\mathrm{VO}_{2 \text { max }}$ similares, podem manter uma maior intensidade de corrida sem que isso incorra em um quadro acidótico. Outro estudo demonstrou que corredores quenianos também apresentam maior economia de corrida do que atletas caucasianos, permitindo com que eles tenham um menor gasto energético para uma dada intensidade de corrida (Weston, Mbambo \& Myburgh, 2000).

Em relação às pesquisas sobre os métodos de treinamento de resistência, vários autores têm sugerido que o treinamento intervalado, em que períodos de atividade de alta intensidade são intercalados por períodos de repouso ativo, é capaz de aumentar tanto o $\mathrm{VO}_{2 \text { máx }}$ quanto o limiar anaeróbio em indivíduos altamente treinados que não conseguem mais aumentar o desempenho usando o método tradicional (i.e. método contínuo) (Billat, 2001; Billat, Demarle, Paiva \& Koralsztein, 2002; Billat, Slawinski, Bocquet, Demarle, Lafitte, Chassaing \& Koralsztein, 2000).

Já a economia de corrida tem sido mais recentemente estudada. Diversos grupos tem se dedicado a investigação dos efeitos do treino de força e potência na melhora da economia de corrida, baseados na premissa de que alterações no componente muscular (e.g. "stiffness" músculo-tendíneo e eficiência na utilização do ciclo alongamento encurtamento) poderiam aumentar a eficiência mecânica da corrida, tornado-a mais econômica. De fato, alguns autores demonstram efeitos positivos do treinamento de força e potência sobre a economia de corrida, criando um novo paradigma de treino para atletas de resistência (Kyrolainen, Kivela, Koskinen, McBride, AnderSen, Takala, Sipila \& Komi, 2003; Paavolainen, Hakkinen, Hamalainen, Nummela \& Rusko, 1999).

\section{Conclusão}

$\mathrm{O}$ alto desempenho físico-esportivo observado nos atletas atuais é fruto da interação de fatores fisiológicos, biomecânicos e psicológicos. Nesta perspectiva, programas de treinamento corretamente elaborados e baseados em princípios científicos são fundamentais na busca constante do rendimento máximo. As ciências do esporte têm contribuído, ainda que de maneira tímida, para o aperfeiçoamento destes programas, melhorando progressivamente a qualidade do processo de treinamento.

Assim, é cada vez mais importante que os profissionais do esporte recebam informações que possibilitem o desenvolvimento completo dos seus atletas. A compreensão dos diferentes componentes que contribuem para um alto desempenho numa determinada modalidade esportiva passa pela aplicação de testes específicos que possam auxiliar na elaboração, implementação, avaliação e reformulação de programas de treinamentos bem como no monitoramento dos progressos obtidos.

O estudo isolado de métodos e meios de aperfeiçoamento das capacidades motoras evoluiu consideravelmente nos últimos 20 anos. A aplicação de novas tecnologias de mensuração e avaliação tem permitido o maior entendimento do desenvolvimento destas capacidades. Porém, a "teoria" do treinamento ainda carece de estudos de caráter mais integrativo envolvendo não somente a combinação simultânea do treinamento de capacidades e habilidades motoras como também dos efeitos dos diferentes modelos de periodização. 


\begin{abstract}
Physical training: scientific and practical considerations

Physical training is an important field of work for the Physical Education and Sport professional. Its main goal is to develop the physical capacity and sports performance of an individual by means of an organized and systematic application of physical exercises. In the past years, the technological progress in the scientific investigation within the different disciplines related to physical training have contributed a great deal towards the accomplishment of such goal. In this manuscript we discuss, from both an academic-scientific and from a practical application point-of-view, the state of the art of the knowledge associated with the physical training evaluation, loading control, loading scheming, and the development of physical abilities. Our goal is to provide a comprehensive overview of the different components of the physical training and how they contribute to the development of the physical performance as well as how to apply these concepts in the elaboration, implementation, and evaluation of physical training programs.
\end{abstract}

UnITRMs: Physical training; Physical and sports performance; Professional practice in Physical Education and Sport.

\title{
Referências
}

ABERNETHY, P.; WILSON, G.; LOGAN, P. Strength and power assessment: issues, controversies and challenges. Sports Medicine, Auckland, v.19, n.6, p.401-17, 1995.

AHTIAINEN, J.P.; PAKARINEN, A.; ALEN, M.; KRAEMER, W.J.; HAKKINEN, K. Short vs. long rest period between the sets in hypertrophic resistance training: influence on muscle strength, size, and hormonal adaptations in trained men. Journal of Strength and Conditioning Research, Champaign, v.19, n.3, p.572-82, 2005.

BACURAU, R.F.; MONTEIRO, G.A.; UGRINOWITSCH, C.; TRICOLI, V.; CABRAL, L.F.; AOKI, M.S. Acute effect of a ballistic and a static stretching exercise bout on flexibility and maximal strength. Journal of Strength and Conditioning Research, Champaign, v.23, n.1, p.304-8, 2009.

BARBANTI, V.J.; TRICOLI, V.; UGRINOWITSCH, C. Relevância do conhecimento científico na prática do treinamento físico. Revista Paulista de Educação Física, São Paulo, v.18, p.101-9, 2004. Número especial.

BASSETT JUNIOR, D.R.; HOWLEY, E.T. Limiting factors for maximum oxygen uptake and determinants of endurance performance. Medicine and Science in Sports and Exercise, Madison, v.32, n.1, p.70-84, 2000.

BATISTA, M.A.; UGRINOWITSCH, C.; ROSCHEL, H.; LOTUFO, R.; RICARD, M.D.; TRICOLI, V.A. Intermittent exercise as a conditioning activity to induce postactivation potentiation. Journal of Strength and Conditioning Research, Champaign, v.21, n.3, p.837-40, 2007.

BEN ABDELKRIM, N.; CASTAGNA, C.; EL FAZAA, S.; EL ATI, J. The effect of players' standard and tactical strategy on game demands in men's basketball. Journal of Strength and Conditioning Research, Champaign, v.24, n.10, p.2652-62, 2010.

BILLAT, L.V. Interval training for performance: a scientific and empirical practice: special recommendations for middle- and long-distance running. Part I: aerobic interval training. Sports Medicine, Auckland, v.31, n.1, p.13-31, 2001.

BILLAT, V.; DEMARLE, A.; PAIVA, M.; KORALSZTEIN, J.P. Effect of training on the physiological factors of performance in elite marathon runners (males and females). International Journal of Sports Medicine, Stuttgart, v.23, n.5, p.336-41, 2002. BILLAT, V.L.; SLAWINSKI, J.; BOCQUET, V.; DEMARLE, A.; LAFITTE, L.; CHASSAING, P.; KORALSZTEIN, J.P. Intermittent runs at the velocity associated with maximal oxygen uptake enables subjects to remain at maximal oxygen uptake for a longer time than intense but submaximal runs. European Journal of Appliel Physiology, Berlin, v.81, n.3, p.188-96, 2000. BISHOP, D. An applied research model for the sport sciences. Sports Medicine, Auckland, v.38, n.3, p.253-63, 2008. BORRESEN, J.; LAMBERT, M.I. The quantification of training load, the training response and the effect on performance. Sports Medicine, Auckland, v.39, n.9, p.779-95, 2009.

BOSCO, C.; BELLI, A.; ASTRUA, M.; TIHANYI, J.; POZZO, R.; KELLIS, S.; TSARPELA, O.; FOTI, C.; MANNO, R.; TRANQUILLI, C. A dynamometer for evaluation of dynamic muscle work. European Journal of Applied Physiology and Occupational Physiology, Berlin, v.70, n.5, p.379-86, 1995.

62 • Rev. bras. Educ. Fís. Esporte, São Paulo, v.25, p.53-65, dez. 2011 N. esp. 
CASTRO, J.; MESQUITA, I. Estudo das implicações do espaço ofensivo nas características do ataque no voleibol masculino de elite. Revista Portuguesa de Ciências do Desporto, Porto, v.8, n.1, p.114-25, 2008.

CIPRIANI, D.; ABEL, B.; PIRRWITZ, D. A comparison of two stretching protocols on hip range of motion: implications for total daily stretch duration.Journal of Strength and Conditioning Research, Champaign, v.17, n.2, p.274-8, 2003. CRONIN, J.B.; McNAIR, P.J.; MARSHALL, R.N. The role of maximal strength and load on initial power production. Medicine and Science in Sports and Exercise, Madison, v.32, n.10, p.1763-9, 2000.

CURRELL, K.; JEUKENDRUP, A.E. Validity, reliability and sensitivity of measures of sporting performance. Sports Medicine, Auckland, v.38, n.4, p.297-316, 2008.

DAVIS, D.S.; ASHBY, P.E.; McCALE, K.L.; McQUAIN, J.A.; WINE, J.M. The effectiveness of 3 stretching techniques on hamstring flexibility using consistent stretching parameters. Journal of Strength and Conditioning Research, Champaign, v.19, n.1, p.27-32, 2005. DE SOUZA, E.; ROSA, L.; PIRES, F.; WILSON, J.; FRANCHINI, E.; TRICOLI, V.; UGRINOWITSCH, C. The acute effects of varying strength exercises bouts on $5 \mathrm{~km}$ running. Journal of Sports Science and Medicine, London, v.10, p.565-70, 2011. DE SOUZA, E.O.; TRICOLI, V.; FRANCHINI, E.; PAULO, A.C.; REGAZZINI, M.; UGRINOWITSCH, C. Acute effect of two aerobic exercise modes on maximum strength and strength endurance. Journal of Strength and Conditioning Research, Champaign, v.21, n.4, p.1286-90, 2007.

FORTEZA, A. Treinamento desportivo: carga, estrutura e planejamento. 2. ed. São Paulo: Phorte, 2006.

FOSTER, C.; DAINES, E.; HECTOR, L.; SNYDER, A.; WELSH, R. Athletic performance in relation to training load. Winconsin Medical Journal, Madison, v.95, p.370-4, 1996.

FOSTER, C.; FLORHAUG, J.A.; FRANKLIN, J.; GOTTSCHALL, L.; HROVATIN, L.A.; PARKER, S.; DOLESHAL, P.; DODGE, C. A new approach to monitoring exercise training. Journal of Strength and Conditioning Research, Champaign, v.15, n.1, p.109-15, 2001.

FRENCH, D.N.; KRAEMER, W.J.; COOKE, C.B. Changes in dynamic exercise performance following a sequence of preconditioning isometric muscle actions. Journal of Strength and Conditioning Research, Champaign, v.17, n.4, p.678-85, 2003. GARDINER, N. Athletics of the Ancient world. Oxford: University Press, 1930.

HIGBIE, E.J.; CURETON, K.J.; WARREN, G.L., 3RD; PRIOR, B.M. Effects of concentric and eccentric training on muscle strength, cross-sectional area, and neural activation. Journal of Applied Physiology, Bethesda, v.81, n.5, p.2173-81, 1996. HILL-HAAS, S.; BISHOP, D.; DAWSON, B.; GOODMAN, C.; EDGE, J. Effects of rest interval during high-repetition resistance training on strength, aerobic fitness, and repeated-sprint ability. Journal of Sports Science, London, v.25, n.6, p.619-28, 2007. HOPKINS, W.G. Measures of reliability in sports medicine and science. Sports Medicine, Auckland, v.30, n.1, p.1-15, 2000. HOUGH, J.P.; PAPACOSTA, E.; WRAITH, E.; GLEESON, M. Plasma and salivary steroid hormone responses of men to highintensity cycling and resistance exercise. Journal of Strength and Conditioning Research, Champaign, v.25, n.1, p.23-31, 2011. ISSURIN, V.B. New horizons for the methodology and physiology of training periodization. Sports Medicine, Auckland, v.40, n.3, p.189-206, 2010.

JONES, A.M.; CARTER, H. The effect of endurance training on parameters of aerobic fitness. Sports Medicine, Auckland, v.29, n.6, p.373-86, 2000.

KISS, M.; BOHME, M. Avaliação de treinamento esportivo In: KISS, M.A.P.D.M. Esporte e exercício: avaliação e prescrição. São Paulo: Roca, 2003. p.3-20.

KRAEMER, W.J.; HAKKINEN, K.; NEWTON, R.U.; NINDL, B.C.; VOLEK, J.S.; McCORMICK, M.; GOTSHALK, L.A.; GORDON, S.E.; FLECK, S.J.; CAMPBELL, W.W.; PUTUKIAN, M.; EVANS, W.J. Effects of heavy-resistance training on hormonal response patterns in younger vs. older men. Journal of Applied Physiology, Bethesda, v.87, n.3, p.982-92, 1999. KYROLAINEN, H.; KIVELA, R.; KOSKINEN, S.; McBRIDE, J.; ANDERSEN, J.L.; TAKALA, T.; SIPILA, S.; KOMI, P.V. Interrelationships between muscle structure, muscle strength, and running economy. Medicine and Science in Sports and Exercise, Madison, v.35, n.1, p.45-9, 2003.

LAMAS, K.; ROSE JUNIOR, D.; SANTANA, F.; ROSTAISER, E.; NEGRETTI, L.; UGRINOWITSCH, C. Space creation dynamics in basketball offense: validation and evaluaton of elite teams. International Journal of Performance Analysis in Sport, Cardiff, v.11, p.71-84, 2011.

LAMAS, L.; BATISTA, M.; FONSECA, R.; PIVETTI, B.; TRICOLI, V.; UGRINOWITSCH, C. Treinamento de potência muscular para membros inferiores: número ideal de repetições em função da intensidade e densidade da carga. Revista da Educação Física/UEM, Maringá, v.21, p.263-270, 2010.

LAMAS, L.; DREZNER, R.; TRICOLI, V.; UGRINOWISTCH, C. Efeito de dois métodos de treinamento no desenvolvimento da força máxima e da potência muscular de membros inferiores. Revista Brasileira de Educaçáo Física e Esporte, São Paulo, v.22, n.3, p.235-45, 2008. 
LAMBERT, M.I.; BORRESEN, J. Measuring training load in sports. International Journal of Sports Physiology and Performance, Champaign, v.5, n.3, p.406-11, 2010.

LARSEN, H.B. Kenyan dominance in distance running. Comparative Biochemistry and Physiology: Molecular \& Integrative Physiology, New York, v.136, n.1, p.161-70, 2003.

MATVEEV, L. Teoria general del entrenamiento deportivo. Barcelona: Editorial Paidotribo, 2001.

McBRIDE, J.M.; McCAUlLEY, G.O.; CORMIE, P.; NUZZO, J.L.; CAVILL, M.J.; TRIPLETT, N.T. Comparison of methods to quantify volume during resistance exercise.Journal of Strength and Conditioning Research, Champaign, v.23, n.1, p.106-10, 2009.

MERO, A.; KOMI, P.V.; GREGOR, R.J. Biomechanics of sprint running: a review. Sports Medicine, Auckland, v.13, n.6, p.376-92, 1992.

MOHR, M.; KRUSTRUP, P.; ANDERSSON, H.; KIRKENDAL, D.; BANGSBO, J. Match activities of elite women soccer players at different performance levels. Journal of Strength and Conditioning Research, Champaign, v.22, n.2, p.341-9, 2008. MOREIRA, A. A eficácia e a heterocronia das respostas de adaptaçáo de basquetebolistas submetidos a diferentes modelos de estruturação da carga de treinamento e competição. 2006. - Universidade Estadual de Campinas, Campinas, 2006. MORITANI, T.; De VRIES, H.A. Neural factors versus hypertrophy in the time course of muscle strength gain. American Journal of Physical Medicine, Baltimore, v.58, n.3, p.115-30, 1979.

MULLIGAN, S.E.; FLECK, S.J.; GORDON, S.E.; KOZIRIS, L.P.; TRIPLETT-McBRIDE, N.T.; KRAEMER, W.J. Influence of resistance exercise volume on serum growth hormone and cortisol concentrations in women. Journal of Strength and Conditioning Research, Champaign, v.10, n.4, p.256-62, 1996.

NAVARRO, F. La resistência. Madri: Gyminos, 1998.

NIEMAN, D.C. Exercise, infection, and immunity. Internatinal Journal of Sports Medicine, Stuttgart, v.15, p.S13141, 1994. Supplement 3.

NISSINEN, M. Kinematic and kinetic analysis of the giant swing on rings. In: KOMI, P.V. Biomechanics VIII-B. Baltimore: University Park Press, 1983. p.781-6.

NUNES, J.A.; CREWTHER, B.T.; UGRINOWITSCH, C.; TRICOLI, V.; VIVEIROS, L.; De ROSE JUNIOR, D.; AOKI, M.S. Salivary hormone and immune responses to three resistance exercise schemes in elite female athletes. Journal of Strength and Conditioning Research, Champaign, v.25, n.8, p.2322-7, 2011.

PAAVOLAINEN, L.; HAKKINEN, K.; HAMALAINEN, I.; NUMMELA, A.; RUSKO, H. Explosive-strength training improves 5-km running time by improving running economy and muscle power. Journal of Applied Physiology, Bethesda, v.86, n.5, p.1527-33, 1999.

PARCELL, A.C.; SAWYER, R.D.; TRICOLI, V.A.; CHINEVERE, T.D. Minimum rest period for strength recovery during a common isokinetic testing protocol. Medicine and Science in Sports and Exercise, Madison, v.34, n.6, p.1018-22, 2002. PAYNE, A.; BARKER, P. Comparison of the take-off forces in the flic flac and the back somersault in gymnastics. In: KOMI, P.V. Biomechanics VIII-B. Baltimore: University Park Press, 1975. p.314-21.

PLATONOV, V. Tratado geral de treinamento desportivo. São Paulo: Phorte, 2008.

RATAMESS, N.A.; ALVAR, B.A.; EVETOCH, T.K.; HOUSH, T.J.; KIBLER, W.B.; KRAEMER, W.J. American College of Sports Medicine position stand: progression models in resistance training for healthy adults. Medicine and Science in Sports and Exercise, Madison, v.41, n.3, p.687-708, 2009.

RHEA, M.R. Synthesizing strength and conditioning research: the meta-analysis. Journal of Strength and Conditioning Research, Champaign, v.18, n.4, p.921-3, 2004.

RICARD, M.D.; UGRINOWITSCH, C.; PARCELL, A.C.; HILTON, S.; RUBLEY, M.D.; SAWYER, R.; POOLE, C.R. Effects of rate of force development on EMG amplitude and frequency. Internatinal Journal of Sports Medicine, Stuttgart, v.26, n.1, p.66-70, 2005.

ROSCHEL, H.; BARROSO, R.; BATISTA, M.; UGRINOWITSCH, C.; TRICOLI, V.; ARSATI, F.; LIMA-ARSATI, Y.B.; ARAUJO, V.C.; MOREIRA, A. Do whole-body vibration exercise and resistance exercise modify concentrations of salivary cortisol and immunoglobulin A? Brazilian Journal of Medicine and Biological Research, Ribeirão Preto, v.44, n.6, p.592-7, 2011. ROSCHEL, H.; UGRINOWISTCH, C.; BARROSO, R.; BATISTA, M.A.; SOUZA, E.O.; AOKI, M.S.; SIQUEIRA-FILHO, M.A.; ZANUTO, R.; CARVALHO, C.R.; NEVES, M.; MELLO, M.T.; TRICOLI, V.Effect of eccentric exercise velocity on akt/mtor/ p70(s6k) signaling in human skeletal muscle. Applied Physiology, Nutrition, and Metabolism, Ottawa, v.36, n.2, p.283-90, 2011. ROWLANDS, A.V.; MARGINSON, V.F.; LEE, J. Chronic flexibility gains: effect of isometric contraction duration during proprioceptive neuromuscular facilitation stretching techniques. Research Quarterly for Exercise and Sport, Washington, v.74, n.1, p.47-51, 2003.

64 • Rev. bras. Educ. Fís. Esporte, São Paulo, v.25, p.53-65, dez. 2011 N. esp. 
SALONIKIDIS, K.; ZAFEIRIDIS, A. The effects of plyometric, tennis-drills, and combined training on reaction, lateral and linear speed, power, and strength in novice tennis players. Journal of Strength and Conditioning Research, Champaign, v.22, n.1, p.182-91, 2008.

SHEPPARD, J.M.; GABBETT, T.J.; STANGANELLI, L.C. An analysis of playing positions in elite men's volleyball: considerations for competition demands and physiologic characteristics. Journal of Strength and Conditioning Research, Champaign, v.23, n.6, p.1858-66, 2009.

SHEPHARD, R.J. Limits to the measurement of habitual physical activity by questionnaires. British Journal of Sports Medicine, London, v.37, n.3, p.197-206, 2003. [discussion 206].

SMILIOS, I.; PILIANIDIS, T.; KARAMOUZIS, M.; TOKMAKIDIS, S.P. Hormonal responses after various resistance exercise protocols. Medicine and Science in Sports and Exercise, Madison, v.35, n.4, p.644-54, 2003.

TAKARADA, Y. Evaluation of muscle damage after a rugby match with special reference to tackle plays. British Journal of Sports Medicine, London, v.37, n.5, p.416-9, 2003.

THACKER, S.B.; GILCHRIST, J.; STROUP, D.F.; KIMSEY JUNIOR, C.D. The impact of stretching on sports injury risk: a systematic review of the literature. Medicine and Science in Sports and Exercise, Madison, v.36, n.3, p.371-8, 2004. TILP, M.; WAGNER, H.; MULLER, E. Differences in 3D kinematics between volleyball and beach volleyball spike movements. Sports Biomechanics, Edinburg, v.7, n.3, p.386-97, 2008.

TOJI, H.; SUEI, K.; KANEKO, M. Effects of combined training loads on relations among force, velocity, and power development. Canadian Journal of Applied Physiology, Champaign, v.22, n.4, p.328-36, 1997.

TRICOLI, V.; LAMAS, L.; CARNEVALE, R.; UGRINOWITSCH, C. Short-term effects on lower-body functional power development: weightlifting vs. vertical jump training programs. Journal of Strength and Conditioning Research, Champaign, v.19, n.2, p.433-7, 2005.

UGRINOWITSCH, C.; BARBANTI, V.; GONÇALVES, A.; PERES, B. Capacidade dos testes isocinéticos em predizer a "performance" no saldo vertical em jogadores de voleibol. Revista Paulista de Educaçáo Física, São Paulo, v.14, n.2, p.172-83, 2000. UGRINOWITSCH, C.; TRICOLI, V.; RODACKI, A.L.; BATISTA, M.; RICARD, M.D. Influence of training background on jumping height. Journal of Strength and Conditioning Research, Champaign, v.21, n.3, p.848-52, 2007. VERKHOSHANSKY, Y. Fundamentals of special strength-training in sport. Livonia: Sportivny, 1986.

WALLACE, L.K.; SLATTERY, K.M.; COUTTS, A.J. The ecological validity and application of the session-RPE method for quantifying training loads in swimming. Journal of Strength and Conditioning Research, Champaign, v.23, n.1, p.33-8, 2009. WEINECK, J. Biologia do esporte. São Paulo: Manole, 1999.

WESTON, A.R.; MBAMBO, Z.; MYBURGH, K.H. Running economy of African and Caucasian distance runners. Medicine and Science in Sports and Exercise, Madison, v.32, n.6, p.1130-4, 2000.

WILLARDSON, J.M.; BURKETT, L.N. The effect of rest interval length on bench press performance with heavy vs. light loads. Journal of Strength and Conditioning Research, Champaign, v.20, n.2, p.396-9, 2006.

WILSON, J.M.; HORNBUCKLE, L.M.; KIM, J.S.; UGRINOWITSCH, C.; LEE, S.R.; ZOURDOS, M.C.; SOMMER, B.; PANTON, L.B. Effects of static stretching on energy cost and running endurance performance. Journal of Strength and Conditioning Research, Champaign, v.24, n.9, p.2274-9, 2010.

YUKTASIR, B.; KAYA, F. Investigation into the long-term effects of static and PNF stretching exercises on range of motion and jump performance. Journal of Bodywork and Movement Therapies, New York, v.13, n.1, p.11-21, 2009. ZAKHAROV, A.; GOMES, A. Ciência do treinamento desportivo. Rio de Janeiro: Palestra Sport, 2003.

\section{Agradecimentos}

Os autores agradecem a Diego Barretti e Fabiano Pinheiro pela contribuição na revisão do texto final deste manuscrito.

ENDEREÇO

Hamilton Rochel

Escola de Educação Física e Esporte - USP

Av. Prof. Mello Moraes, 65

05508-030 - São Paulo - SP - BRASIL

e-mail:hars@usp.br 